\subsection{USING COHORTS TO STUDY LIFECOURSE EPIDEMIOLOGY}

\author{
Chair: Prof Cyrus Cooper, UK \\ Discussant: Prof John Frank, UK \\ 02-2.1 MAXIMISING THE RETURN FROM COHORT STUDIES
}

doi:10.1136/jech.2011.142976a.52

${ }^{1} \mathrm{~A}$ Leyland, ${ }^{*}{ }^{2} \mid$ White, ${ }^{1} \mathrm{~S}$ Harding, ${ }^{2} \mathrm{~S}$ Seaman, ${ }^{3} \mathrm{C}$ Booker. ${ }^{1} \mathrm{MRC} \mid \mathrm{CSO}$ Social and Public Health Sciences Unit, Glasgow, UK; ${ }^{2}$ MRC Biostatistics Unit, Cambridge, UK; ${ }^{3}$ Institute of Social and Economic Research, University of Essex, UK

Introduction Cohort studies are important for understanding the aetiology underlying differences in disease incidence. Selective attrition is problematic as those at greater risk of ill-health are more likely to drop out, resulting in homogenous study populations with limited generalisability. Selective attrition may also bias estimates of association. For the benefits of cohort studies to be realised efforts must be made to minimise attrition and statistical methods for the analysis of studies with missing data must be developed to minimise bias.

Methods We describe work examining methods used to maintain participation in cohort studies, identify best practice for reducing attrition and investigate methodologies suitable for the analysis of cohort studies with attrition under different circumstances.

Results A literature search identified factors associated with minimising attrition including study design, recruitment procedures, incentives and retention methods utilised. A questionnaire including such factors was sent to 32 UK-based cohort studies; 25 (78\%) returned a questionnaire. Analysis suggested that no one method is most effective, rather it is the combination of methods and study setting which may dictate the overall retention of study participants.

Work with various cohort studies resulted in consideration of the appropriateness of methods for missing data. This has led to a review of inverse probability weighting (IPW) focusing on how and when to use it appropriately. The motivation for combining IPW and multiple imputation, and the theoretical justification for doing so, has been examined. A final development concerns the use of IPW when predictors of missingness are themselves missing.

\section{2-2.2 MEASUREMENT AND MODELLING OF FUNCTIONAL TRAJECTORIES ACROSS THE LIFE COURSE}

doi:10.1136/jech.2011.142976a.53

${ }^{1} \mathrm{R}$ Hardy, ${ }^{* 2} \mathrm{~F}$ Matthews, ${ }^{1} \mathrm{D}$ Kuh, ${ }^{3} \mathrm{D}$ Lawlor, ${ }^{4} \mathrm{~A}$ A Sayer, ${ }^{5} \mathrm{M}$ Benzeval. ${ }^{1} \mathrm{M} R \mathrm{C}$ Unit for Lifelong Health and Ageing, London, UK; ${ }^{2}$ MRC Biostatistics Unit, Cambridge, UK; ${ }^{3}$ MRC Centre for Causal Analyses in Translational Epidemiology, Bristol, UK; ${ }^{4} M R C$ Lifecourse Epidemiology Unit, Southampton, UK; ${ }^{5}$ MRC |CSO Social and Public Health Sciences Unit, Glasgow, UK

Introduction Epidemiological studies are increasingly including measures of function as well as disease status but there are no guiding principles on which to base the choice of measures. The overall aim of this project is to develop recommendations for the measurement of function and the modelling of functional trajectories within and across cohort studies. Continuous and normally distributed measures of biological function, repeated over time, provide dynamic tools for studying the biological imprint of physical and social exposures. Signs of impaired function may act as intermediate markers of underlying disease processes, failure to reach developmental potential, or accelerated ageing, and offer opportunities for early intervention. There is currently no single study which has repeated measures of function from birth to old age. The best description of life course functional trajectories currently will come from the pooling of data from cohorts spanning the whole age range. Harmonisation of measures of function is required to facilitate this, while development of the statistical methods for combining trajectories is necessary.

Methods and Results We illustrate progress towards the ultimate objective of modelling the life course trajectories of cardiovascular, physical and cognitive function using data from multiple cohorts, and investigating risk factors influencing their shape. Using blood pressure as an example, we present results from the modelling of the longitudinal trajectories in multiple UK cohorts of different ages. We discuss issues relating to the comparability of measures both within and between studies, and the development of statistical methods for the synthesis of trajectories across cohorts.

\section{2-2.3 USING GENETIC VARIANTS AS INSTRUMENTAL VARIABLES IN COHORT STUDIES}

doi:10.1136/jech.2011.142976a.54

D Lawlor. ${ }^{*}$ MRC Centre for Causal Analyses in Translational Epidemiology, Bristol, UK

Observational epidemiological studies suffer from many potential biases, from confounding and from reverse causation, and this limits their ability to robustly identify causal associations. In other observational sciences, notably econometrics, the use of instrumental variable approaches has been one approach to strengthening causal inferences in non-experimental situations. The use of germline genetic variants as instruments for modifiable (non-genetic) risk factors is one form of instrumental variables analysis that can be implemented within observational epidemiological studies. The method has been referred to as "Mendelian randomisation", and can be considered as analogous to randomised controlled trials. This presentation will briefly define Mendelian randomisation and instrumental variable analysis; demonstrate how the instrumental variable approach differs from multivariable regression (the more common approach to dealing with confounding in observational analysis) and discuss the potential and limitations of genetic variants as instrumental variables. Recent methodological developments that might be used to address some of the limitations will also be discussed.

\section{2-2.4 USING COHORTS TO STUDY LIFECOURSE EPIDEMIOLOGY: THE MRC LIFECOURSE EPIDEMIOLOGY UNIT}

doi:10.1136/jech.2011.142976a.55

\section{Cooper. ${ }^{*}$ MRC Lifecourse Epidemiology Unit, Southampton, UK}

The MRC Lifecourse Epidemiology Unit maintains a large number of internationally unique cohort resources permitting evaluation of the developmental origins of chronic disease. Some of these comprise population-based samples of adults in whom detailed phenotyping has been undertaken; DNA has been stored; and birth/ infant records have been retrieved (eg, include the Hertfordshire Cohort Study, the Helsinki Cohort Study, and the Delhi Cohort Study). Others comprise more contemporary mother-offspring studies (eg, the Southampton Women's Survey and the Pune Maternal Nutrition Study). The purpose of the Unit's research is to elucidate important, preventable causes of common chronic disorders and their complications. In particular, these cohorts permit study of the interplay of causes acting at different stages of the lifecourse, from before conception through to old age. Through an understanding of the causes of these disorders and evaluation of 